\title{
Evolution of regorafenib from bench to bedside in colorectal cancer: Is it an attractive option or merely a "me too" drug?
}

This article was published in the following Dove Press journal: Cancer Management and Research

\section{Gaurav Goel \\ Division of Hematology-Oncology, University of Pittsburgh School of Medicine, Pittsburgh, PA, USA}

\section{Video abstract}

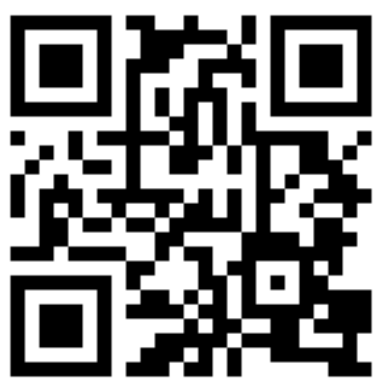

Point your SmartPhone at the code above. If you have a QR code reader the video abstract will appear. Or use: http://youtu.be/ZMhWfX9;594
Correspondence: Gaurav Goel Division of Hematology-Oncology, University of Pittsburgh School of Medicine, 2000 Oxford Drive, Suite 500,

Pittsburgh, PA 15102, USA

$\mathrm{Tel}+|4| 283 \mid 1320$

Fax + 4 4I2 83I 9748

Email ggoel.uky@gmail.com
Abstract: Colorectal cancer (CRC) is a major public health problem in the United States with an estimated 50,260 deaths in 2017. Over the past two decades, several agents have been approved by the US Food and Drug Administration (FDA) for the treatment of patients with metastatic CRC (mCRC). Regorafenib (BAY 73-4506) is a small-molecule multikinase inhibitor that was approved for the treatment of $\mathrm{mCRC}$ in 2012. This agent is a novel oral diphenylureabased multikinase inhibitor that is active against several angiogenic receptor tyrosine kinases (RTKs; VEGFR-1, VEGFR-2, VEGFR-3, TIE-2), oncogenic RTKs (c-KIT, RET), stromal RTKs (PDGFR-B, FGFR-1), and intracellular signaling kinases (c-RAF/RAF-1, BRAF, BRAF ${ }^{\mathrm{V} 600 \mathrm{E}}$ ). Preclinical studies have documented its broad-spectrum activity against different solid tumor types including CRC. Phase I studies showed that it had an acceptable safety profile in advanced refractory mCRC. A subsequent Phase III trial (CORRECT) demonstrated significant clinical efficacy of regorafenib in patients with refractory or advanced $\mathrm{mCRC}$, which eventually led to its FDA approval for the treatment of mCRC in September 2012. However, the drug was associated with significant toxicity in clinical practice when administered at the approved doses, which necessitated a thorough reassessment of its dosing schedule and toxicity profile. This review summarizes the development of regorafenib from the initial preclinical studies to the Phase III trials and critically examines the current clinical space occupied by regorafenib in the treatment of $\mathrm{mCRC}$, at 5 years after its initial FDA approval.

Keywords: regorafenib, multikinase inhibitor, angiogenesis, colorectal cancer, colon cancer, stivarga, BAY 73-4506

\section{Introduction}

Colorectal cancer (CRC) is a major public health problem in the United States and globally. In the United States, it is the second leading cause of cancer mortality. In 2017 , it is estimated that over 50,000 deaths will be attributed to this disease. ${ }^{1}$ When metastatic disease is diagnosed, CRC is associated with poor prognosis, with 5 -year survival rates in the 5\%-8\% range. Chemotherapy has been the traditional approach for patients with metastatic CRC (mCRC). Over the past two decades, several agents such as bevacizumab, cetuximab, panitumumab, and ziv-aflibercept have been approved by the US Food and Drug Administration (FDA) for the treatment of mCRC. Newer agents including regorafenib, TAS-102, ramucirumab, and anti-PD1 immunotherapy have been recently added to this list during the past 5 years. $^{2,3}$ Regorafenib is a small-molecule multikinase inhibitor that was approved in 2012 for patients with mCRC who are refractory to or intolerant of standard chemotherapy. 
It is now well established that multiple signaling pathways work in a coordinated fashion to regulate tumor growth, survival, angiogenesis, and invasion/migration. Tumor angiogenesis has been one of the major targets for the development of new anticancer agents with great success. Tumor angiogenesis is mediated by several established growth factors and receptor pathways such as VEGFRs, tyrosine kinase with immunoglobulin and epidermal growth factor homology domain 2 (TIE-2), fibroblast growth factor receptor (FGFR), and platelet-derived growth factor receptor (PDGFR). Among these various growth factor receptor-mediated pathways, the VEGF signaling pathway has been considered to be the most important regulator of tumor angiogenesis. ${ }^{4}$ It also mediates the autocrine effects on the survival, migration, and invasion of tumor cells. This has led to the development of several targeted agents aimed at inhibiting VEGF signaling. Currently known approaches include inhibition of ligand binding to the target VEGF receptors by antibodies (bevacizumab and ziv-aflibercept), blockade of ligand-VEGFR binding by anti-VEGFR-2 antibody such as ramucirumab, and inhibition of intracellular receptor tyrosine kinases (RTKs) of VEGFRs with receptor tyrosine kinase inhibitors (TKIs), such as regorafenib. ${ }^{5}$

The various VEGF ligands, VEGF-A, VEGF-B, VEGFC, VEGF-D, and placental growth factor (P1GF), bind to their respective cognate receptors VEGFR-1, VEGFR-2, and
VEGFR-3, and induce activation of VEGFR signaling. It is widely believed that the tumor angiogenic effects of VEGF are mediated, in large part, through VEGFR-2. TIE-2 is another important regulator of tumor angiogenesis that is mainly expressed in endothelial cells. ${ }^{6}$ It modulates maturation of immature vessels upon binding to its ligands including angiopoietin-1 (Ang1), angiopoetin-2 (Ang2), VEGF, and FGF. ${ }^{7}$

Regorafenib (BAY 73-4506; Bayer Schering Pharma AG, Berlin, Germany) is an oral small-molecule multikinase inhibitor that is active against several angiogenic RTKs (VEGFR-1, VEGFR-2, VEGFR-3, TIE-2), oncogenic RTKs (c-KIT, RET), stromal RTKs (PDGFR-B, FGFR1), and intracellular signaling kinases (c-RAF/RAF-1, BRAF, BRAF ${ }^{\mathrm{V} 600 \mathrm{E}}$; Figure 1). In 2012, regorafenib was approved by the US FDA for the treatment of patients with $\mathrm{mCRC}$ who have progressed on standard chemotherapies. This review will summarize the development of regorafenib from the initial preclinical studies to the current clinical era in the treatment of mCRC.

\section{Preclinical development}

Regorafenib (BAY 73-4506) is an oral multikinase inhibitor with a diphenylurea backbone, 4-[4-(\{[4-chloro3 -(trifluoromethyl)phenyl]carbamoyl \}amino )3-fluorophenoxy]- $N$-methylpyridine-2-carboxamide. ${ }^{8}$ This agent originally came from a drug discovery program with urea class compounds following the development of

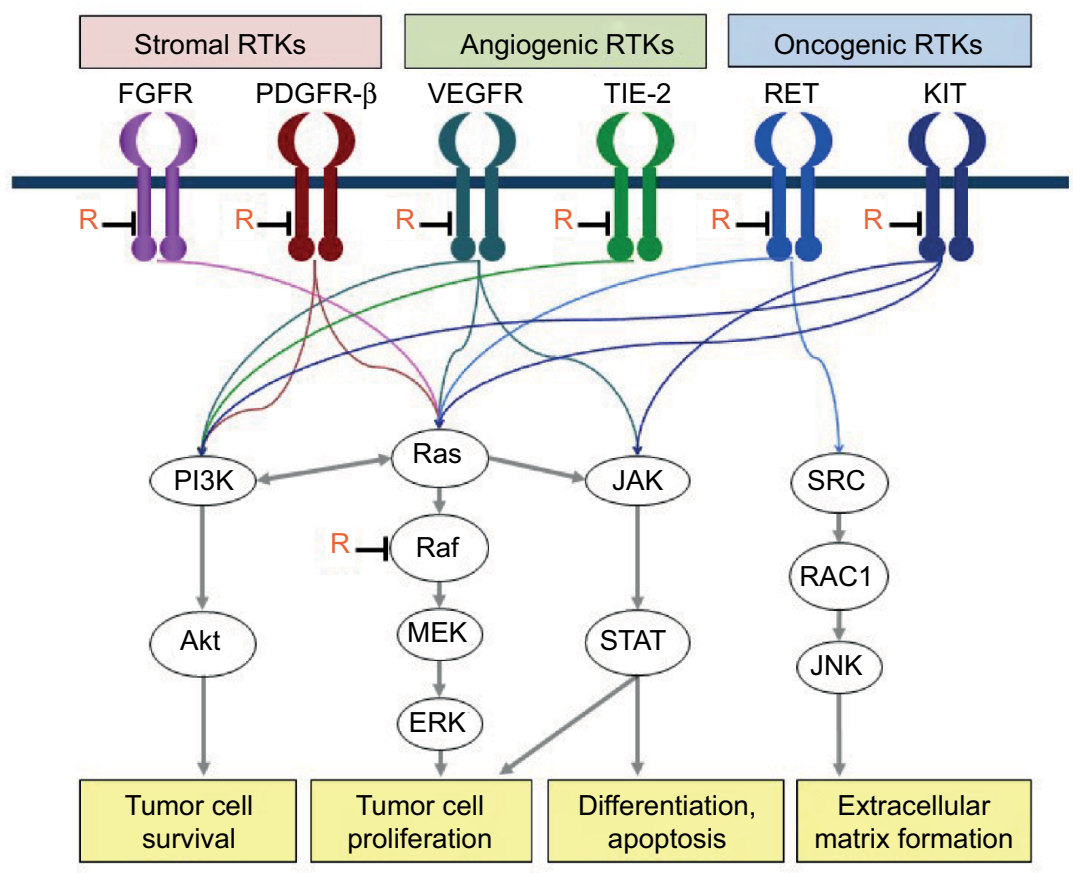

Figure I Regorafenib inhibits multiple tyrosine kinases.

Note: $R$ denotes regorafenib.

Abbreviations: FGFR, fibroblast growth factor receptor; PDGFR, platelet-derived growth factor receptor; RTKs, receptor tyrosine kinases; VEGFR, vascular endothelial growth factor receptors. 
sorafenib, a successful prototype of diphenylurea compounds discovered in the 1990s. ${ }^{8}$ In contrast to sorafenib, regorafenib has a fluorine in the center phenyl ring (Figure 2A and 2B). This additional structural modification of regorafenib results in a similar but distinct biochemical profile when compared with sorafenib (Table 1). ${ }^{8-10}$

\section{In vitro biochemical assays}

In vitro biochemical assays revealed that regorafenib inhibits several angiogenic RTKs (VEGFR-1, murine VEGFR-2, murine VEGFR-3, and TIE-2) with 50\% inhibitory concentration $\left(\mathrm{IC}_{50}\right)$ values ranging from 4.2 to $311 \mathrm{nM} / \mathrm{L}$ (Table 2$){ }^{8}$ Oncogenic RTKs including c-KIT and RET were also inhibited by regorafenib with $\mathrm{IC}_{50}$ values ranging from 1.5 to $7 \mathrm{nM} / \mathrm{L} .{ }^{8}$ In addition, stromal RTKs including PDGFR- $\beta$ and FGFR1 were inhibited with $\mathrm{IC}_{50}$ values ranging from 22 to $202 \mathrm{nM} / \mathrm{L}$. Intracellular signaling kinases including c-RAF/RAF-1, wild-type BRAF, and mutant BRAF ${ }^{\mathrm{V} 600 \mathrm{E}}$ were inhibited with $\mathrm{IC}_{50}$ values ranging from 2.5 to 28 $\mathrm{nM} / \mathrm{L}{ }^{8}$ Of note, regorafenib at concentrations, as high as $1 \mu \mathrm{M} / \mathrm{L}$, did not inhibit a wide range of kinases, including kinases associated with the epidermal growth factor receptor (EGFR) family, protein kinase $\mathrm{C}$ family, cyclin-dependent kinases, and kinases associated with insulin and insulin-like growth factor receptor, c-MET, MEK, ERK1/2, and AKT. ${ }^{8}$ In terms of metabolism, the drug is exclusively metabolized in the liver via cytochrome P3A4 and uridine diphosphateglucuronosyltransferase 1A9, which increases the risk of drug-induced liver injury. ${ }^{11}$ The two active metabolites of regorafenib include $\mathrm{M}-2$ ( $N$-oxide metabolite, BAY 75-7495) and M-5 ( $N$-oxide/ $N$-desmethyl metabolite, BAY 81-8752).

\section{Cellular phosphorylation assays}

Cellular phosphorylation assays showed that regorafenib is a potent inhibitor of multikinases including VEGFR-2 ${ }^{8}$ Regorafenib is a potent inhibitor of VEGFR-2 autophosphorylation in NIH-3T3/VEGFR-2 cells with an $\mathrm{IC}_{50}$ value of $3 \mathrm{nM} / \mathrm{L}$. Similarly, regorafenib potently inhibited TIE-2 autophosphorylation in vanadate-stimulated Chinese hamster ovary-TIE-2 cells with an $\mathrm{IC}_{50}$ value of $31 \mathrm{nM} / \mathrm{L}$. Regorafenib inhibited PDGFR- $\beta$ autophosphorylation after stimulation with PDGF$\mathrm{BB}$ in human aortic smooth muscle cells (HAoSMCs) with an $\mathrm{IC}_{50}$ value of $90 \mathrm{nM} / \mathrm{L}$. Regorafenib also inhibited FGFR signaling in $\mathrm{MCF}-7$ human breast cancer cells stimulated with FGF10 with an $\mathrm{IC}_{50}$ value of $\sim 200 \mathrm{nM} / \mathrm{L}$.

Regorafenib inhibited the MAPK signaling pathway, as measured by pERK1/2 enzyme-linked immunosorbent assay (ELISA), in various tumor models carrying mutations in $K R A S$ or $B R A F$ with $\mathrm{IC}_{50}$ values of $43-380 \mathrm{nM} / \mathrm{L}$. It also potently inhibited mutant RTKs including $\mathrm{KIT}^{\mathrm{K} 642 \mathrm{E}}$ and $\mathrm{RET}^{\mathrm{C} 634 \mathrm{~W}}$ with $\mathrm{IC}_{50}$ values of $10-20 \mathrm{nM} / \mathrm{L} .^{8}$ This inhibitory effect on the respective KIT and RET activity helps to explain the clinical activity of regorafenib in patients with gastrointestinal stromal tumors and a subset of medullary thyroid carcinomas. In the cellular phosphorylation assays, the M-2 and M-5 metabolites inhibited VEGFR-2, TIE-2, c-KIT, and BRAF with $\mathrm{IC}_{50}$ values similar to that of regorafenib (Table 3). ${ }^{12,13}$

\section{In vitro cell proliferation assays}

Regorafenib displays strong in vitro antiproliferative effects on growth factor-stimulated vascular and tumor cell lines

Table I Comparison of in vitro biochemical kinase inhibition by regorafenib and sorafenib

\begin{tabular}{|c|c|c|}
\hline Kinase target & $\begin{array}{l}\text { Regorafenib }^{8} \\
\left(\mathrm{IC}_{50}[\mathrm{nM} / \mathrm{L}]\right)\end{array}$ & $\begin{array}{l}\text { Sorafenib } \\
\left(\mathrm{IC}_{50}[\mathrm{nM} / \mathrm{L}]\right)\end{array}$ \\
\hline VEGFR-I & 13 & N/A \\
\hline VEGFR-2 & 4.2 & 15 \\
\hline VEGFR-3 & 46 & 20 \\
\hline TIE-2 & 311 & N/A \\
\hline PDGFR- $\beta$ & 22 & 57 \\
\hline FGFR I & 202 & 580 \\
\hline BRAF & 28 & 22 \\
\hline $\mathrm{BRAF}^{\mathrm{V} 600 \mathrm{E}}$ & 19 & 38 (V599E) \\
\hline
\end{tabular}

Note: VEGFR-2/3 are murine VEGFRs.

Abbreviations: FGFR, fibroblast growth factor receptor; $\mathrm{IC}_{50}, 50 \%$ inhibitory concentration; N/A, not available; PDGFR, platelet-derived growth factor receptor; TIE-2, tyrosine kinase with immunoglobulin and epidermal growth factor homology domain 2; VEGFR, vascular endothelial growth factor receptors.
A

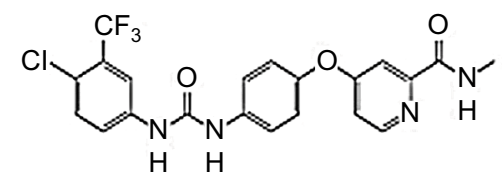

B

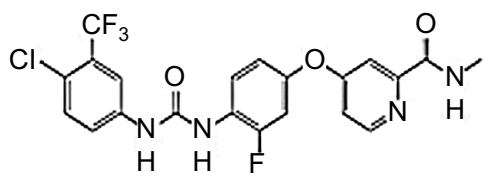

Figure 2 Chemical structure of Sorafenib (A) and Regorafenib (B).

Note: The only structural difference between these two drugs is that regorafenib has a fluorine in the center phenyl ring. 
(Table 4). In vitro cell proliferation assays demonstrated that regorafenib inhibited the proliferation of VEGF ${ }^{165}$ stimulated human umbilical vascular endothelial cells (HuVECs) with an $\mathrm{IC}_{50}$ value of $\sim 3 \mathrm{nM} / \mathrm{L} .{ }^{8}$ Regorafenib inhibited the proliferation of FGF2-stimulated HuVECs and PDGF-BB-stimulated HAoSMCs with $\mathrm{IC}_{50}$ values of 127 and $146 \mathrm{nM}$, respectively. ${ }^{8}$ These data are consistent with in vitro biochemical assays, which showed strong inhibition of VEGFR-2 by regorafenib. Regorafenib potently inhibits VEGFR-2 HuVECs and VEGFR-3 in lymphatic endothelial cells (LECs) with $\mathrm{IC}_{50}$ values of $4-16 \mathrm{nM} / \mathrm{L} .{ }^{14}$ Moreover, it inhibited the phosphorylation of ERK and AKT kinases in LECs with $\mathrm{IC}_{50}$ values of $4-16 \mathrm{nM} / \mathrm{L} .^{14}$

Regorafenib inhibited the growth of SW620 (KRAS G12V) and Colo-205 (a human CRC cell line harboring $B R A F^{V 600 E}$ mutation) cell lines with $\mathrm{IC}_{50}$ values in the range of 970$3270 \mathrm{nM} / \mathrm{L} .{ }^{8} \mathrm{Using}$ an in vitro proliferation assay, regorafenib inhibited the proliferation of 19 out of 25 human colon cancer cell lines with $\mathrm{IC}_{50}$ values ranging from 2,600 to 10,000 $\mathrm{nM} / \mathrm{L}$, which is comparable to the maximum concentration $\left(\mathrm{C}_{\max }\right)$ of regorafenib observed in plasma from patients. ${ }^{14,15}$ Of note, no correlation was observed between regorafenibmediated antiproliferative effects and the mutational status of the cell lines including mutations in $K R A S$ and $B R A F^{14}$

Table 2 In vitro biochemical activity of regorafenib against various kinases

\begin{tabular}{|c|c|c|}
\hline Kinase category & Kinases & $\begin{array}{l}\text { Regorafenib }^{8} \\
\left(\mathrm{IC}_{50}[\mathrm{nM} / \mathrm{L}]\right)\end{array}$ \\
\hline Angiogenic RTKs & $\begin{array}{l}\text { VEGFR-I, } \\
\text { VEGFR-2, } \\
\text { VEGFR-3, TIE-2 }\end{array}$ & $4.2-311$ \\
\hline Oncogenic RTKs & c-KIT, RET & $1.5-7$ \\
\hline Stromal RTKs & PDGFR- $\beta$, FGFR I & $22-202$ \\
\hline Intracellular signaling & c-RAF/RAF-I, wild-type & $2.5-28$ \\
\hline kinases & $\mathrm{BRAF}$, and mutant $\mathrm{BRAF} \mathrm{F}^{\mathrm{V} 600 \mathrm{E}}$ & \\
\hline
\end{tabular}

Abbreviations: FGFR, fibroblast growth factor receptor; $\mathrm{IC}_{50}, 50 \%$ inhibitory concentration; PDGFR, platelet-derived growth factor receptor; RTKs, receptor tyrosine kinases; TIE-2, tyrosine kinase with immunoglobulin and epidermal growth factor homology domain 2; VEGFR, vascular endothelial growth factor receptor.

\section{Tumor xenograft models}

Regorafenib exhibited the in vivo antitumor activity in several human tumor xenograft models. The growth of Colo-205 in a mouse xenograft model was effectively inhibited by regorafenib in the dose range of $10-100 \mathrm{mg} / \mathrm{kg}$, achieving tumor growth inhibition of about $75 \%$ at day 14 at the dose level of $10 \mathrm{mg} / \mathrm{kg}^{8}$

As a single agent, regorafenib displayed somewhat modest antitumor effects in several patient-derived xenograft (PDX) CRC models. ${ }^{14}$ However, the combination of regorafenib and irinotecan led to a statistically significant delay in tumor growth compared with irinotecan alone in oxaliplatin-refractory PDX CRC models. ${ }^{14}$ Regorafenib treatment significantly reduced tumor vessel areas and the total number of tumor vessels determined by CD31 staining in some PDX CRC models. ${ }^{14}$ However, this antiangiogenic effect of regorafenib was not demonstrated in PDX CRC models, which were refractory to treatment with regorafenib. This finding suggests that the antiangiogenic effect of regorafenib contributes to the inhibition of tumor growth in PDX CRC models. ${ }^{14}$

Sajithlal et al reported a significant synergistic antitumor activity between MK2206, an AKT inhibitor, and regorafenib in an HCT116 colon cancer xenograft model. ${ }^{16}$ Tumor-

Table 4 In vitro antiproliferation activity of regorafenib against vascular and tumor cell lines

\begin{tabular}{|c|c|}
\hline Cell lines & $\begin{array}{l}\text { Regorafenib } 8,14 \\
\left(\mathrm{IC}_{50}[\mathrm{nM} / \mathrm{L}] \pm \mathrm{SD}\right)\end{array}$ \\
\hline VEGF $^{165}$-stimulated HuVECs & $2.6 \pm 0.8$ \\
\hline FGF2-stimulated HuVECs & $127 \pm 13$ \\
\hline PDGF-BB-stimulated HAoSMCs & $146 \pm 1 \mid 4$ \\
\hline VEGFR-2-stimulated HuVECs & $4-16$ \\
\hline VEGFR-3-stimulated LECs & $4-16$ \\
\hline SW620 (KRAS $\left.{ }^{G 12 V}\right)$, colon & $967 \pm 287$ \\
\hline Colo-205, colon & 3269 \\
\hline
\end{tabular}

Abbreviations: FGF, fibroblast growth factor; HAoSMCs, human aortic smooth muscle cells; HuVECs, human umbilical vascular endothelial cells; $\mathrm{IC}_{50}, 50 \%$ inhibitory concentration; LECs, lymphatic endothelial cells; PDGF, platelet-derived growth factor; VEGFR, vascular endothelial growth factor receptor.

Table 3 Pharmacologic activity of regorafenib, $M-2$, and $M-5$ in cellular kinase phosphorylation assays s.,13 $^{12}$

\begin{tabular}{|c|c|c|c|}
\hline Kinase, cell line & $\begin{array}{l}\text { Regorafenib } \\
\left(I_{50}[n M / L] \pm S D\right)\end{array}$ & $\begin{array}{l}\text { Metabolite M-2 } \\
\left(\mathrm{IC}_{50}[\mathrm{nM} / \mathrm{L}] \pm \mathrm{SD}\right)\end{array}$ & $\begin{array}{l}\text { Metabolite M-5 } \\
\left(\mathrm{IC}_{50}[\mathrm{nM} / \mathrm{L}] \pm \mathrm{SD}\right)\end{array}$ \\
\hline VEGFR-2, NIH-3T3 & 40 & 30 & 20 \\
\hline TIE-2, CHO & $31 \pm 9$ & $66 \pm 35$ & $180 \pm 0$ \\
\hline c-KIT wild type, M07e & 23 & 13 & 110 \\
\hline C-KIT ${ }^{\mathrm{K} 642 \mathrm{E}}, \mathrm{GIST} 882$ & $17 \pm 4$ & $4 \pm 2$ & $N / A$ \\
\hline $\mathrm{BRAF}^{\mathrm{V} 600 \mathrm{E}}, \mathrm{RAT}-\mathrm{I}$ & 69 & 21 & 27 \\
\hline
\end{tabular}

Abbreviations: $\mathrm{CHO}$, Chinese hamster ovary; GIST, gastrointestinal stromal tumors; $\mathrm{IC}_{50}$, inhibitory concentration; N/A, not available; TIE-2, tyrosine kinase with immunoglobulin and epidermal growth factor homology domain 2; VEGFR-2, vascular endothelial growth factor receptor 2. 
bearing mice were treated with regorafenib $25 \mathrm{mg} / \mathrm{kg}$ and MK2206 $40 \mathrm{mg} / \mathrm{kg}$ daily for 3 days.

\section{Phase I and II clinical studies}

The safety and tolerability of regorafenib was initially evaluated by a German group in a Phase I study. ${ }^{17}$ This study was performed using the standard " $3+3$ " design, and the eligibility criteria included patients with advanced solid tumors that had progressed after standard therapies. Regorafenib was administered in 28-day treatment cycles using a schedule of 21 days on/7 days off. Oral solution formulation of regorafenib was administered in cohorts at dose levels of 10-120 mg. For dose levels $>120 \mathrm{mg}$, a coprecipitate tablet formulation was administered. Patients were evaluated in eight different dose cohorts, with regorafenib $220 \mathrm{mg}$ once daily being the highest dose evaluated. CRC was the most common tumor type $(30 \%)$ evaluated in this study. ${ }^{17,18}$ Two of 12 patients at the dose level of $160 \mathrm{mg}$ experienced dose-limiting toxicities in cycle 1 , resulting in a dose reduction. The dose of 160 $\mathrm{mg}$ coprecipitate tablets once a day in a schedule of 21 days on/7 days off repeated every 28 days was therefore determined to be the maximum tolerated dose or the recommended phase 2 dose (RP2D) for single-agent regorafenib. The most common adverse events (AEs) included voice changes (55\%), hand-foot-skin reaction (HFSR; 40\%), mucositis (36\%), diarrhea (32\%), and hypertension (HTN; 30\%). The most common grade 3/4 AEs included HFSR (19\%), HTN (11\%), diarrhea (8\%), and rash/desquamation (6\%). With respect to clinical efficacy, 32 patients $(60 \%)$ had stable disease (SD), while 3 patients (CRC, renal cell carcinoma, and osteogenic sarcoma) showed a partial response (PR). Pharmacokinetic (PK) assessment revealed that the area under the curve (AUC) target exposure level of $13 \mathrm{mg} \mathrm{h} / \mathrm{L}$ (from preclinical models) was achieved at the $30 \mathrm{mg}$ dose level. A similar exposure was observed at steady state for regorafenib and its metabolites M-2 and M-5. With the oral solution, systemic exposure of regorafenib increased in a dose-dependent manner up to the $60 \mathrm{mg}$ dose level, with no further increase seen when the dose was increased to $120 \mathrm{mg}$. This agent has a terminal half-life $\left(t_{1 / 2}\right)$ on the order of 20-40 hours, which resulted in its accumulation in plasma after multiple doses.

Regorafenib was further evaluated in patients with $\mathrm{mCRC}$ in the dose-expansion portion of this Phase I trial. ${ }^{15}$ A total of 38 patients with mCRC were enrolled, which included 15 patients in the dose-escalation phase and 23 patients in the dose-expansion phase. These patients had previously received a median of four lines of systemic chemotherapy. Regorafenib was administered at a dose of $160 \mathrm{mg}$ daily for 21 days fol- lowed by rest for 7 days, repeated every 28 days. The median duration of regorafenib treatment at $160 \mathrm{mg}$ daily dose was 49 days (range $8-280$ days). The most common side effects included HFSR (61\%), fatigue (50\%), voice changes (34\%), anorexia (24\%), and diarrhea (24\%). The most common grade $3 / 4$ AEs included HFSR (32\%), fatigue (11\%), HTN (11\%), and rash/desquamation (5\%). Among the 27 evaluable patients, 1 patient (4\%) achieved PR and 19 patients (70\%) achieved SD with a total disease control rate (DCR) of $74 \%$. The median progression-free survival (PFS) was 107 days. The steady-state PK data showed a similar plasma exposure of regorafenib and its metabolites, $M-2$ and $M-5$. The $t_{1 / 2}$ of M-2 and regorafenib was similar (25 hours versus 26-28 hours), while M-5 had a longer $t_{1 / 2}$ of 51-64 hours.

Another Phase I dose-escalation study evaluated continuous regorafenib administered once a day without any rest periods. ${ }^{19}$ The dose cohorts ranged from 20 to $140 \mathrm{mg} /$ day. A total of 38 patients with advanced solid tumors were enrolled in this study: CRC (16\%), thyroid cancer (13\%), adenoid cystic carcinoma (13\%), and head and neck cancer (13\%). The RP2D of regorafenib was determined as $100 \mathrm{mg}$ once a day using this continuous dosing schedule without any rest periods. In general, the drug was well tolerated, and the most frequent AEs were rash/desquamation (50\%), HFSR (32\%), fatigue $(32 \%)$, extremity pain $(29 \%)$, mucositis $(24 \%)$, and diarrhea $(21 \%)$. The most common grade $3 / 4$ AEs were HFSR (11\%), extremity pain (5\%), diarrhea (3\%), and rash/ desquamation (3\%). Two patients $(6 \%)$ achieved PR (one with pancreatic neuroendocrine carcinoma and the other with periorbital squamous cell carcinoma) and 22 patients (61\%) had SD. PK assessment showed that the steady-state plasma levels of regorafenib increased less than proportionally from 20 to $100 \mathrm{mg}$ and did not increase significantly from 100 to $140 \mathrm{mg}$ when dosed daily. ${ }^{19}$

Sunakawa et al reported the results of a Phase I multicenter study, which evaluated the safety, PK, and efficacy of regorafenib in Japanese patients with chemorefractory solid tumors. ${ }^{20} \mathrm{~A}$ total of 15 patients were enrolled in this study, and the most common tumor site was pancreas (six ductal adenocarcinomas and one neuroendocrine tumor). Patients received regorafenib $160 \mathrm{mg}$ orally once daily for 21 days in 28-day cycles. Patients received a median of two cycles (range 1-21), and the duration of treatment was 2.1 months (range 0.9-20.1). The most frequent side effects included HFSR (67\%), diarrhea (67\%), hypophosphatemia (53\%), and aspartate aminotransferase (AST) elevation (53\%). The most common grade 3/4 AEs included hypophosphatemia (27\%), lymphopenia (27\%), HFSR (135), and AST/alanine 
aminotransferase elevation (13\%). One patient with pancreatic neuroendocrine tumor experienced a PR, and seven patients had SD. With respect to clinical pharmacology, the mean systemic exposure of regorafenib, M-2, and M-5 was significantly lower in Japanese patients when compared with European patients. However, the range of regorafenib exposure was similar to that observed in the European trials.

Schultheis et al reported the results of a Phase Ib study, which evaluated the combination of regorafenib with either FOLFOX (5-fluorouracil [5-FU] $400 \mathrm{mg} / \mathrm{m}^{2}$ IV bolus followed by 5 -FU $2,400 \mathrm{mg} / \mathrm{m}^{2}$ IV continuous infusion over 46 hours, folinic acid $400 \mathrm{mg} / \mathrm{m}^{2} \mathrm{IV}$, and oxaliplatin $85 \mathrm{mg} /$ $\mathrm{m}^{2} \mathrm{IV}$ ) or FOLFIRI (5-FU $400 \mathrm{mg} / \mathrm{m}^{2}$ IV bolus followed by 5 -FU 2,400 $\mathrm{mg} / \mathrm{m}^{2}$ IV continuous infusion over 46 hours, folinic acid $400 \mathrm{mg} / \mathrm{m}^{2} \mathrm{IV}$, and irinotecan $180 \mathrm{mg} / \mathrm{m}^{2} \mathrm{IV}$ ) in the first- or second-line treatment of patients with mCRC. ${ }^{21}$ The primary objective of this study was to evaluate the safety and toxicity of regorafenib in combination with FOLFOX or FOLFIRI, and to determine the effect of regorafenib on the PK of FOLFOX and FOLFIRI. A total of 45 evaluable patients were enrolled in the study ( $\mathrm{N}=25$ in FOLFOX arm; $\mathrm{N}=20$ in FOLFIRI arm). Regorafenib $160 \mathrm{mg}$ once a day was administered orally on days 4-10 and 18-24 of each cycle of FOLFOX or FOLFIRI. The incidence of any grade AEs was similar between the FOLFOX and FOLFIRI arms. Grade 3/4 AEs were observed in 12 patients (27\%) and included one treatment-related death (hepatic toxicity). The most common AEs leading to dose modifications of regorafenib included neutropenia $(27 \%)$, mucositis (18\%), HFSR $(18 \%)$, and leukopenia (11\%). In terms of clinical efficacy, 7 out of 38 evaluable patients achieved a PR (4 patients in the FOLFOX arm and 3 patients in the FOLFIRI arm), and 26 patients had SD (14 patients in the FOLFOX arm and 12 patients in the FOLFIRI arm). The median duration of disease control was similar in the FOLFOX (123 days) and FOLFIRI (126 days) arms. PK analysis revealed a potential drug-drug interaction between regorafenib and irinotecan, as there was an increased exposure of irinotecan and its active metabolite $\mathrm{SN}-38$ when regorafenib was administered sequentially after FOLFIRI. However, no such drug-drug interaction was observed between regorafenib and oxaliplatin.

Argilés et al presented the results of the Phase II study evaluating the efficacy and safety of regorafenib in combination with modified FOLFOX6 (mFOLFOX6) in the first-line treatment of patients with mCRC (CORDIAL trial). ${ }^{22}$ Patients with $\mathrm{mCRC}(\mathrm{N}=54)$ received $\mathrm{mFOLFOX6}$ on days 1 and 15 and regorafenib $160 \mathrm{mg}$ orally once a day on days $1-4$ and
18-24, repeated in 28-day cycles. In cases of mFOLFOX6 discontinuation, regorafenib was administered as a single agent at the dose of $160 \mathrm{mg}$ orally once a day in a schedule of 21 days on/7 days off. The primary endpoint of the study was overall response rate (ORR), and the secondary endpoints included overall survival (OS), PFS, DCR, duration of response (DOR), duration of SD, and safety. The ORR was $43.9 \%$ and the DCR was $85.4 \%$. The median PFS of this combination regimen was 8.5 months $(95 \%$ CI $7.4-11.3$ months), and the median duration of SD was 7.6 months. The most common regorafenib-related grade 3/4 AEs included lipase elevation (19\%), HTN (17\%), diarrhea (13\%), and hypophosphatemia (11\%).

\section{Phase III clinical trials}

The CORRECT study was a Phase III trial to evaluate the efficacy of regorafenib in mCRC patients whose tumor had progressed after all approved standard therapies. ${ }^{23}$ This trial was a randomized, placebo-controlled, double-blind study involving 114 centers in 16 countries across four different continents. Patients with $\mathrm{mCRC}(\mathrm{N}=760)$ who had progressed on all available standard therapies or discontinued therapy due to unacceptable toxicity were randomized in a 2:1 ratio to best supportive care (BSC) plus either regorafenib $(\mathrm{N}=505)$ or placebo ( $\mathrm{N}=255)$. Regorafenib was administered at a dose of $160 \mathrm{mg}$ orally once a day for 21 days followed by 7 days of rest in 28-day cycles. The primary endpoint of this study was OS, and the secondary endpoints included PFS, ORR, DCR, and safety. The tertiary endpoints included DOR, healthrelated quality of life (HR-QoL), and health utility values. Most of the baseline characteristics were balanced in both the study arms. KRAS mutations were found in $54 \%$ of patients in the regorafenib arm and $62 \%$ of patients in the placebo arm. The median OS was 6.4 months in the regorafenib arm and 5 months in the placebo arm (one-sided $P=0.0052$ ). The median PFS was 1.9 and 1.7 months, respectively, in the two arms. PR was achieved in five patients in the regorafenib arm and in one patient in the placebo arm. DCR (PR plus SD assessed at least 6 weeks after randomization) was $41 \%$ in the regorafenib arm and $15 \%$ in the placebo arm. The most frequent AEs in the regorafenib arm were fatigue (47\%), HFSR (47\%), and diarrhea (34\%), and the most frequent grade $3 / 4$ AEs were HFSR (17\%), fatigue (10\%), diarrhea (7\%), HTN $(7 \%)$, and rash/desquamation (6\%). The findings from this study led to the US FDA approval of regorafenib in September 2012 for the treatment of patients with $\mathrm{mCRC}$ who have been previously treated with fluoropyrimidine, oxaliplatin, 
and irinotecan-based chemotherapy, an anti-VEGF therapy, and an anti-EGFR therapy (if $K R A S$ wild type). In a post hoc analysis of the CORRECT trial data, regorafenib was shown to have similar efficacy in both Japanese and non-Japanese subpopulations. ${ }^{24}$

The CONCUR study was another randomized, doubleblind, placebo-controlled, Phase III trial of regorafenib that was specifically designed to assess the efficacy of the drug in Asian patients. ${ }^{25}$ Patients with refractory mCRC from China, Hong Kong, South Korea, Taiwan, and Vietnam were randomized in a 2:1 ratio to receive BSC plus either regorafenib $(\mathrm{N}=136)$ or placebo $(\mathrm{N}=68)$. Regorafenib was administered at $160 \mathrm{mg}$ orally once a day in a schedule of 21 days on/7 days off. The primary endpoint of OS was significantly better in the regorafenib arm than in the placebo arm (median OS 8.8 months versus 6.3 months; one-sided $P=0.00016$ ). The most frequent grade 3/4 AEs in the regorafenib arm included HFSR (16\%), HTN (11\%), hyperbilirubinemia (7\%), and hypophosphatemia (7\%).

The activity of regorafenib has also been evaluated in two single-arm, open-label studies (REBECCA and CONSIGN) that were performed in a real-world setting. The REBECCA study was based on a French compassionate program that examined the safety and effectiveness of regorafenib for mCRC patients. ${ }^{26}$ Patients were identified from the database of the French ATU (Temporary Authorization for Use) between December 2012 and October 2013, and 500 patients were included in this analysis. The primary endpoint of this study was OS, and the secondary endpoints included the duration of regorafenib therapy, PFS, safety, and dose intensity of regorafenib therapy. About $10 \%$ of the study population had a performance status (PS) of ECOG $\geq 2$, and $18 \%$ of the patients received regorafenib at the initial dose of $<160 \mathrm{mg} /$ day $(12 \%$ at $120 \mathrm{mg} /$ day and $6 \%$ at $\leq 80 \mathrm{mg} /$ day). The AEs from the REBECCA cohort seemed less frequent than those reported within the CORRECT trial (grade 3/4 AEs, 32\% versus 54\%). However, this difference could also be a result of underreporting in the REBECCA study, due to the retrospective design of the study. Among patients treated at a starting dose of $160 \mathrm{mg}(\mathrm{N}=407)$, there were less dose modifications in the REBECCA cohort than in the CORRECT trial (47\% versus $76 \%$ ). Although some patients from the REBECCA cohort had worse PS and were not treated with the recommended starting dose of regorafenib (160 mg/day), the outcome of patients in the REBECCA study was in the same range as that from the CORRECT trial (PFS, 2.7 months in the REBECCA versus 1.9 months in the CORRECT; OS, 5.5 months versus 6.4 months).
The Phase IIIb CONSIGN study was a prospective, multicenter, expanded-access study that provided regorafenib to patients with chemorefractory $\mathrm{mCRC}$ prior to market authorization. ${ }^{27}$ The primary objective of the study was safety. The safety analysis included 2,864 patients assigned to treatment between April 2012 and December 2013. The grade 3/4 treatmentemergent, drug-related AEs were observed in $57 \%$ patients, and included HTN (15\%), HFSR (14\%), fatigue (13\%), diarrhea (5\%), and hypophosphatemia (5\%). Treatment discontinuation occurred in $9 \%$ of the patients due to drug-related AEs. The estimated median PFS of the cohort was 2.7 months.

Table 5 summarizes the key aspects of all Phase III clinical trials (completed and ongoing) involving regorafenib in patients with $\mathrm{mCRC}$.

\section{Special considerations regarding regorafenib use Tolerability}

One of the main challenges with regorafenib therapy at the current dosing schedule (160 mg/day, 21 days on/7 days off) is that it is associated with a relatively high incidence of AEs and poor tolerance in clinical practice, which results in frequent dose reductions and interruptions.

Safety analysis of the CORRECT trial showed that the incidence of regorafenib-related AEs including HFSR, fatigue, HTN, and rash/desquamation peaked during the first treatment cycle and gradually tapered to lower levels with subsequent cycles, while the incidence of diarrhea remained relatively constant throughout the treatment course. ${ }^{28}$ Dose adjustments were most frequent during cycles 2 and 3, and the dose density of regorafenib stabilized from cycle 4 onward.

Falcone et al analyzed the pattern of regorafenib dose modifications in the CORRECT trial. ${ }^{29}$ Patients randomized to the regorafenib arm stayed on treatment for a median duration of 7.3 weeks and received $78.9 \%$ of the planned regorafenib dose (mean, $147.1 \mathrm{mg}$ ). Only $57 \%$ of patients in the regorafenib arm received an actual daily dose of 160 mg throughout the study. Dose modifications were required in majority (76\%) of patients in the regorafenib arm. Dose reductions and interruptions in regorafenib were reported in $20 \%$ and $70 \%$ of patients, respectively. These data suggest that the starting dose of regorafenib $160 \mathrm{mg}$ may, in fact, be too high. The most frequent side effect leading to the dose modification of regorafenib was HFSR, which was responsible for dose reduction in $18 \%$ of patients and dose interruption in $19 \%$ of patients. 
Table 5 Summary of Phase III clinical trials evaluating regorafenib in patients with $\mathrm{mCRC}$

\begin{tabular}{|c|c|c|c|c|c|}
\hline Trial identifier & $\begin{array}{l}\text { No. of } \\
\text { patients }\end{array}$ & Study arms & Endpoints & $\begin{array}{l}\text { OS (months); } \\
\text { HR (95\% CI) }\end{array}$ & $\begin{array}{l}\text { PFS (months); } \\
\text { HR ( } 95 \% \mathrm{Cl})\end{array}$ \\
\hline \multicolumn{6}{|l|}{ CORRECT } \\
\hline $\begin{array}{l}\text { NCTOII } 03323 \text { (randomized } \\
\text { Phase III study) }\end{array}$ & 760 & $\begin{array}{l}\text { Regorafenib }(N=505) \text { versus } \\
\text { placebo }(N=255)\end{array}$ & $\begin{array}{l}\text { Pri: OS } \\
\text { Sec: PFS, RR, DCR } \\
\text { QoL: EORTC-QLQ-C30 }\end{array}$ & $\begin{array}{l}6.4 \text { versus } \\
5.0 ; 0.77 \\
(0.64-0.94)\end{array}$ & $\begin{array}{l}1.9 \text { versus } 1.7 ; 0.48 \\
(0.42-0.58)\end{array}$ \\
\hline \multicolumn{6}{|l|}{ CONCUR } \\
\hline $\begin{array}{l}\text { NCTOI } 584830 \text { (randomized } \\
\text { Phase III study) }\end{array}$ & 204 & $\begin{array}{l}\text { Regorafenib }(\mathrm{N}=136) \text { versus } \\
\text { placebo }(\mathrm{N}=68)\end{array}$ & $\begin{array}{l}\text { Pri: OS } \\
\text { Sec: PFS, RR, DCR } \\
\text { QoL: EORTC- } \\
\text { QLQ-C30 }\end{array}$ & $\begin{array}{l}8.8 \text { versus } \\
6.3 ; 0.55 \\
(0.40-0.77)\end{array}$ & $\begin{array}{l}3.2 \text { versus } 1.7 ; 0.31 \\
(0.22-0.44)\end{array}$ \\
\hline \multicolumn{6}{|l|}{ REBECCA } \\
\hline $\begin{array}{l}\text { NCT023I0477 (observational } \\
\text { cohort study) } \\
\text { CONSIGN }\end{array}$ & 500 & Regorafenib & $\begin{array}{l}\text { Pri: OS } \\
\text { Sec: PFS }\end{array}$ & 5.5 & 2.7 \\
\hline $\begin{array}{l}\text { NCT0I538680 (expanded- } \\
\text { access Phase Illb study) } \\
\text { REGARD }\end{array}$ & 2872 & Regorafenib & $\begin{array}{l}\text { Pri: Safety } \\
\text { Sec: PFS }\end{array}$ & Not reported & 2.7 \\
\hline $\begin{array}{l}\text { NCT0I8533I9 (ongoing } \\
\text { Phase III study) }\end{array}$ & 100 & Regorafenib & Pri: Safety, PFS & Not reported & Not reported \\
\hline COTEZO IMblaze370 & & & & & \\
\hline $\begin{array}{l}\text { NCT02788279 (ongoing, } \\
\text { randomized Phase III study) }\end{array}$ & 360 & $\begin{array}{l}\text { Cobimetinib+atezolizumab } \\
\text { versus atezolizumab versus } \\
\text { regorafenib }\end{array}$ & $\begin{array}{l}\text { Pri: OS } \\
\text { Sec: PFS, DOR, safety } \\
\text { QoL: EORTC- } \\
\text { QLQ-C30, EQ-5D-5L }\end{array}$ & Not reported & Not reported \\
\hline
\end{tabular}

Abbreviations: DCR, disease control rate; DOR, duration of response; HR, hazard ratio; $\mathrm{mCRC}$, metastatic colorectal cancer; OS, overall survival; PFS, progression-free survival; Pri, primary; QoL, quality of life; RR, response rate; Sec, secondary.

TAS-102 is a combination of thymidine-based nucleoside analog (trifluridine) and thymidine phosphorylase inhibitor (tipiracil), and this drug is also currently approved for the treatment of mCRC refractory to standard therapies. A retrospective study showed that both regorafenib and TAS102 have comparable efficacy but different toxicity profiles in patients with mCRC. ${ }^{30}$ The most frequent grade 3 or higher AEs were hepatotoxicity and hand-foot syndrome in the regorafenib group, and neutropenia in the TAS-102 group. Another recently published multicenter observational study (REGOTAS) from Japan compared the efficacy of regorafenib versus TAS-102 in patients with chemorefractory mCRC ${ }^{31}$ The propensity score adjusted analysis in the REGOTAS study showed that OS was similar between the two groups (7.9 months in the regorafenib group and 7.4 months in the TAS-102 group). However, discontinuation of treatment due to treatment-related AEs was higher in the regorafenib group than in the TAS-102 group (24\% versus $7 \%$ ).

Given the significant tolerability issues with regorafenib on the approved dosing regimen, some have suggested that the benefit provided by regorafenib may not be clinically meaningful. A lower starting dose of regorafenib with an incremental approach based on AEs has also been suggested by some experts to improve the drug tolerability in clinical practice. ${ }^{32,33}$

\section{Quality of life}

The initial analysis of HR-QoL from the CORRECT trial suggested that the regorafenib therapy was not associated with impaired overall HR-QoL. ${ }^{34}$ One of the major weaknesses of patient-reported outcomes research is that missing data could be due to deterioration of general health condition from disease progression or AEs. Chang et al reported the effects of regorafenib on HR-QoL in the CORRECT trial by incorporating the data of tumor progression and survival into the analysis of HR-QoL to reduce the bias induced by missing data due to worsening of health from disease progression or AEs. ${ }^{35}$ The Cox model analysis showed that the regorafenib treatment was associated with a significant reduction in the hazard ratio (HR) for both global health status (HR 0.77 ; 95\% CI $0.65-0.91$ ) and physical function (HR 0.74; 95\% CI 0.62-0.88) after adjustment for stratification factors and other covariates. These results suggest that treatment with regorafenib was, indeed, associated with a reduced risk of deterioration in HR-QoL by $>20 \%$ in comparison with placebo alone. 


\section{Cost-effectiveness}

Regorafenib offers modest survival benefit, and therefore, its cost-effectiveness is currently a subject of much debate. Seal et al studied the potential cost-effectiveness of regorafenib for the treatment of patients with mCRC. Their analysis showed that patients treated with regorafenib had improved OS in comparison with those on BSC alone ( 0.75 versus 0.60 years), but regorafenib treatment was also associated with higher lifetime costs than BSC alone $(\$ 97,700$ versus $\$ 59,494) .{ }^{36}$ Goldstein et al also studied the cost-effectiveness of regorafenib in the third-line setting for mCRC patients, using a Markov model ${ }^{37}$ Treatment with regorafenib provided an additional 0.13 life-years at a cost of $\$ 40,000$, resulting in an incremental cost-effectiveness ratio of $\$ 900,000$ per quality-adjusted life-years. The cost of this benefit is considered high by most experts in the field; however, it may be considered justifiable by some given the high unmet need for effective therapies in patients with mCRC. Nevertheless, the cost-effectiveness of the drug can potentially be improved by utilizing biomarkerdriven patient selection and value-based pricing.

\section{Radiographic assessment of tumor response}

It has been postulated that the response to multikinase inhibitors cannot be accurately evaluated using the conventional dimensional criteria such as RECIST. ${ }^{38,39}$ Antiangiogenic agents can cause cavitation in lung tumors, which is thought to be a consequence of central necrosis due to inhibition of blood supply to the tumor. ${ }^{40}$ Ricotta et al have demonstrated that in mCRC patients treated with regorafenib, development of cavitation in lung metastases is associated with response to therapy ${ }^{41,42}$ On the other hand, a smaller study by Lim et al did not find similar significant association between lung metastaseis cavitation and survival..$^{43}$ Treatment with regorafenib can also induce a decrease in tumor density in the liver metastases, but this change is not predictive of clinical outcome. ${ }^{42,43}$ Therefore, at the present time, RECIST 1.1 and change in the sum of target lesion diameters remain as the preferred methods to assess response to regorafenib in mCRC patients..$^{42}$ Lung metastasis cavitation has shown the promising activity as a potential new radiological biomarker and deserves further evaluation.

\section{Identification of biomarkers}

The clinical efficacy data from the CORRECT trial established the role of regorafenib in the treatment of patients with mCRC. However, real-life treatment with regorafenib is associated with significant toxicity leading to frequent treatment discontinuation. ${ }^{44-48}$ An analysis of the Kaplan-Meier curves for PFS in the CORRECT trial suggests that there may be a distinct subgroup of mCRC patients who are more likely to be responsive to regorafenib treatment. ${ }^{23}$ This finding has generated significant interest in identifying potential biomarkers predictive of regorafenib efficacy. When successful, this could help to define patients who are most appropriate for regorafenib treatment. However, the nonspecific inhibitory activity of regorafenib across a wide range of angiogenic, oncogenic, stromal, and intracellular signaling kinases makes the identification of potential predictive biomarkers rather difficult and necessitates the application of a more comprehensive approach. Novel putative biomarkers are currently being evaluated in both the tumor tissue and the peripheral blood samples that were obtained from mCRC patients treated with regorafenib.

\section{Dynamic contrast-enhanced magnetic resonance imaging}

Dynamic contrast-enhanced magnetic resonance imaging (DCE-MRI) is a noninvasive functional imaging modality that can be used to evaluate tumor hemodynamics, and it has been well studied in both preclinical and clinical models. ${ }^{49,50}$

Wilhelm et al demonstrated the pharmacodynamic effect of regorafenib on the tumor vasculature in vivo in a rat GS9L glioblastoma xenograft model by using DCE-MRI with Gadomer-17. ${ }^{8}$ Tumor-bearing rats were treated with a single dose of regorafenib orally at $10 \mathrm{mg} / \mathrm{kg}$. Regorafenib treatment resulted in a significant decrease in tumor perfusion and extravasation of the contrast agent, as measured by the AUC of the initial 360 seconds $\left(\mathrm{iAUC}_{360}\right)$, and this effect persisted for up to 2 days $(P<0.01)$. Inhibition of tumor growth was also noted in this rat GS9L glioblastoma xenograft model as evidenced by the change in volume of intramuscular tumors pre- and posttreatment with regorafenib.

Mross et al measured tumor perfusion before and during regorafenib treatment using DCE-MRI in the Phase I study of regorafenib. ${ }^{17}$ The AUC of the initial 60 seconds (iAUC ${ }_{60}$ ) was used as the DCE-MRI endpoint. The DCE-MRI assessments before and after 21 days of multiple regorafenib dosing (at dose levels of $\geq 120 \mathrm{mg}$ ) showed an average decrease in tumor perfusion by $\geq 40 \%$.

Strumberg et al evaluated single-agent regorafenib in 38 patients with $\mathrm{mCRC}$ who were enrolled in the initial Phase I study during the dose-escalation and dose-expansion phases. ${ }^{15}$ Tumor perfusion analysis was measured by DCE-MRI before and after multiple regorafenib doses. The iAUC $_{60}$ was used 
as the DCE-MRI endpoint. The tumor perfusion measured by DCE-MRI decreased in most patients, and the median ratio of $\mathrm{iAUC}_{60}$ between baseline and day 21 of cycle 2 was 0.507 (range $0.031-1.53 ; \mathrm{N}=20$ ). Unfortunately, no significant association between $\mathrm{iAUC}_{60}$ and PFS $(P=0.74)$ was observed.

Eschbach et al studied the role of contrast-enhanced ultrasound (CEUS) with VEGFR-2-targeted microbubbles to monitor the functional and molecular therapy effect of regorafenib in rat HT-29 CRC xenografts. ${ }^{51}$ Tumor-bearing rats received daily treatment with regorafenib or placebo (10 mg/kg) for 1 week. In CEUS, tumor perfusion was assessed during an early vascular phase (wash-in area under the curve) and VEGFR-2-specific binding during a late molecular phase. The changes in tumor perfusion noted on CEUS were correlated with DCE-MRI parameters (plasma flow and plasma volume) and immunohistochemical staining (for VEGFR-2, CD31, Ki-67, and TUNEL). The CEUS perfusion parameters decreased significantly with regorafenib therapy and showed significant correlations $(P<0.05)$ with DCE-MRI parameters and immunohistochemistry.

\section{Levels of CA 19-9 and cytokines}

A retrospective study of $121 \mathrm{mCRC}$ patients treated with regorafenib evaluated the role of serum biochemical parameters such as carcinoembryonic antigen and carbohydrate antigen 19-9 (CA 19-9) as predictive biomarkers. ${ }^{52}$ In both univariate and multivariate analyses, early CA 19-9 decrease was identified as an independent predictive factor of response to regorafenib therapy. These results require further validation using external data sets prior to clinical use.

Plasma concentrations of VEGF and soluble VEGFR-2 (sVEGFR-2) have been evaluated by quantitative ELISA before and during regorafenib treatment. ${ }^{17}$ The plasma sVEGFR-2 concentration decreases during regorafenib treatment in a dosedependent manner. In contrast, plasma concentrations of VEGF increased over 21 days of regorafenib treatment and returned to baseline levels during the 7-day off treatment. In a biomarker analysis of $54 \mathrm{mCRC}$ patients, 11 angiogenic and inflammatory serum cytokines were evaluated as predictors of regorafenib efficacy and toxicity. ${ }^{53}$ A decrease in VEGF-A levels on day 21 was associated with better PFS. The chemokine ligand 5 (CCL5) levels $\leq$ cutoff value $(59,959 \mathrm{pg} / \mathrm{mL})$ at baseline were also associated with better PFS and OS.

\section{Gene mutations and polymorphisms}

An exploratory biomarker study was conducted using DNA isolated from plasma samples obtained from 503
mCRC patients enrolled in the CORRECT trial. ${ }^{54}$ Mutations in $K R A S, P I K 3 C A$, and $B R A F$ were identified using BEAMing (beads, emulsion, amplification, and magnetics) technology. ${ }^{55,56}$ BEAMing of plasma DNA detected $K R A S$ mutations in $69 \%, P I K 3 C A$ mutations in $17 \%$, and $B R A F$ mutations in $3 \%$ patients. Correlative subgroup analyses demonstrated that treatment with regorafenib was associated with clinical benefit in both $K R A S$ wild-type and mutant subgroups that were identified by plasma BEAMing technology (PFS: wild-type $K R A S$, HR $0.52,95 \%$ CI 0.35-0.76; mutant $K R A S$, HR $0.51,95 \%$ CI $0.40-0.65 ; P=0.74)$. Similar results were noted for $P I K 3 C A$ wild-type and mutant subgroups (PFS: wild-type $P I K 3 C A$, HR $0.50,95 \%$ CI $0.40-0.63$; mutant PIK3CA, HR 0.54, 95\% CI 0.32-0.89; $P=0.85)$.

Polymorphisms in ABCG2 and SLCO1B genes have been studied for their potential role as predictors of adverse drug reactions to regorafenib. In a preliminary study of 37 Japanese patients treated with regorafenib, the absence of SLCO1B $1 * 1 b$ allele was associated with increased treatment-related toxicity. ${ }^{57}$

\section{p53-upregulated modulator of apoptosis (PUMA)}

PUMA is a BH3-only Bcl-2 family member and plays a critical role in the regulation of apoptosis by antagonizing antiapoptotic Bcl-2 family members, including Bcl-XL, and activating proapoptotic members Bax and Bak. ${ }^{58}$ PUMA is transcriptionally activated by $\mathrm{p} 53$ and initiates apoptosis in response to DNA damage. PUMA can also be induced in a p53-independent pathway including nuclear factor $\mathrm{kB}$ (NF$\mathrm{kB}$ ) pathway by a variety of non-genotoxic stimuli. Chen et al showed that regorafenib treatment induced upregulation of PUMA in human CRC cells, which is correlated with apoptosis induction. ${ }^{58}$ In wild-type p53 HCT116 human colon cancer cell lines, regorafenib treatment induced PUMA protein and mRNA expression in a dose- and timedependent manner, and the peaks of PUMA protein and mRNA induction were detected at 24 hours of regorafenib treatment and at $40 \mu \mathrm{M} / \mathrm{L} .{ }^{58}$ Regorafenib-induced apoptosis was more prominent in a CRC cell line with intact PUMA expression than those with defective PUMA expression, suggesting that PUMA may play a critical role in mediating apoptosis induced by regorafenib in human CRC cell lines. The upregulation of PUMA by regorafenib is mediated by NF-kB pathway. In CRC cell lines with intact PUMA expression, regorafenib potentiated upregulation of PUMA induced by 5-FU, oxaliplatin, or cetuximab, suggesting potential synergistic interaction. These data suggest that intact PUMA 
expression could be a predictive biomarker of response to regorafenib treatment.

\section{FDG-PET-based metabolic response assessment}

RegARd-C is a multicenter prospective study evaluating the role of early FDG-PET to identify non-benefiters from regorafenib treatment (160 mg/day, 21 days on/7 days off) based on PET evaluation at baseline and at day 14 of the first treatment course. OS is the primary endpoint and will be correlated with metabolic response assessed by FDG-PET and epigenetic alterations. The target patient accrual is 105 evaluable patients with pretreated $\mathrm{mCRC}$.

\section{Conclusion}

Regorafenib is an orally administered, multitargeted kinase inhibitor that has a single-agent activity in mCRC. The CORRECT trial demonstrated that regorafenib significantly increased OS, PFS, and DCR versus placebo in patients with chemorefractory $\mathrm{mCRC},{ }^{19}$ and based on the results of this study, regorafenib was approved by the US FDA in September 2012 for the treatment of patients with refractory $\mathrm{mCRC}$.

One of the main challenges with regorafenib therapy at the currently approved dosing schedule $(160 \mathrm{mg} /$ day, 21 days on/7-days off) is that it is associated with a relatively high incidence of AEs and poor tolerance in clinical practice, which results in frequent dose reductions and interruptions. Post hoc safety analyses of the CORRECT trial data suggest that timely dose modifications were effective for the management of treatment-emergent AEs. In addition, regorafenibrelated AEs were most frequent with the initial one to two cycles with gradually tapering in incidence and severity in later cycles. It is highly recommended to closely monitor for any regorafenib-related AEs, especially during the initial one to two cycles of regorafenib treatment, and to perform dose modifications as necessary. It would also be helpful to study if an alternative dosing schedule of regorafenib such as continuous daily dosing would be better tolerated than intermittent dosing.

As only a small fraction of patients with advanced or refractory $\mathrm{mCRC}$ derive true clinical benefit from regorafenib, there is a significant unmet need to identify biomarkers that could identify which patients would benefit from regorafenib treatment. Further efforts should be focused on the development of such predictive biomarkers for regorafenib therapy.

\section{Disclosure}

The author reports no conflicts of interest in this work.

\section{References}

1. Siegel RL, Miller KD, Jemal A. Cancer Statistics, 2017. CA Cancer J Clin. 2017;67(1):7-30.

2. Goel G, Chauhan A, Hosein PJ. Ramucirumab: a novel antiangiogenic agent in the treatment of metastatic colorectal cancer. Curr Colorectal Cancer Rep. 2016;12(5):232-240.

3. Myint ZW, Goel G. Role of modern immunotherapy in gastrointestinal malignancies: a review of current clinical progress. $J$ Hematol Oncol. 2017;10(1):86.

4. Ferrara N. Vascular endothelial growth factor as a target for anticancer therapy. Oncologist. 2004;9(Suppl 1):2-10.

5. Ellis LM, Hicklin DJ. VEGF-targeted therapy: mechanisms of antitumour activity. Nat Rev Cancer. 2008;8(8):579-591.

6. Augustin HG, Koh GY, Thurston G, Alitalo K. Control of vascular morphogenesis and homeostasis through the angiopoietin-Tie system. Nat Rev Mol Cell Biol. 2009;10(3):165-177.

7. Yancopoulos GD, Davis S, Gale NW, Rudge JS, Wiegand SJ, Holash J. Vascular-specific growth factors and blood vessel formation. Nature. 2000;407(6801):242-248.

8. Wilhelm SM, Dumas J, Adnane L, et al. Regorafenib (BAY 73-4506): a new oral multikinase inhibitor of angiogenic, stromal and oncogenic receptor tyrosine kinases with potent preclinical antitumor activity. Int J Cancer. 2011;129(1):245-255.

9. Wilhelm SM, Carter C, Tang L, et al. BAY 43-9006 exhibits broad spectrum oral antitumor activity and targets the RAF/MEK/ERK pathway and receptor tyrosine kinases involved in tumor progression and angiogenesis. Cancer Res. 2004;64(19):7099-7109.

10. Wilhelm S, Adnane 1, Hirth-Dietrich C, Ehrlich P, Lynch M. Preclinical characterization of BAY 73-4506: a kinase inhibitor with broad spectrum antitumor activity targeting oncogenic and angiogenic kinases. $A A C R$ Meeting Abstracts.2007;6(11):B260.

11. Sacre A, Lanthier N, Dano H, et al. Regorafenib induced severe toxic hepatitis: characterization and discussion. Liver Int.2016;36(11):1590-1594

12. Zopf D, Scholz A, Fichtner I, Becker M, Müller T, Kissel M. Regorafenib (BAY 73-4506): a broad spectrum tumor deactivator with high combinability potential and antimetastasis activity. AACR; Cancer Res. 2011;71(8 Suppl):Abstract nr 4262.

13. Zopf D, Fichtner I, Bhargava A, et al. Pharmacologic activity and pharmacokinetics of metabolites of regorafenib in preclinical models. Cancer Med. 2016;5(11):3176-3185.

14. Schmieder R, Hoffmann J, Becker M, et al. Regorafenib (BAY 73-4506): antitumor and antimetastatic activities in preclinical models of colorectal cancer. Int J Cancer. 2014;135(6):1487-1496.

15. Strumberg D, Scheulen ME, Schultheis B, et al. Regorafenib (BAY 73-4506) in advanced colorectal cancer: a phase I study. Br JCancer 2012;106(11):1722-1727.

16. Sajithlal GB, Hamed HA, Cruickshanks N, et al. Sorafenib/regorafenib and phosphatidyl inositol 3 kinase/thymoma viral proto-oncogene inhibition interact to kill tumor cells. Mol Pharmacol. 2013;84(4): $562-571$.

17. Mross K, Frost A, Steinbild S, et al. A phase I dose-escalation study of regorafenib (BAY 73-4506), an inhibitor of oncogenic, angiogenic, and stromal kinases, in patients with advanced solid tumors. Clin Cancer Res. 2012;18(9):2658-2667.

18. Hedbom S, Steinbild S, Frost A, et al. Phase I study of BAY 73-4506, a multikinase inhibitor, administered for 21 days on/7 days off in patients with advanced solid tumors. J Clin Oncol. 2007;25(18 Suppl):3593.

19. Shimizu T, Tolcher AW, Patnaik A, et al. Phase I dose-escalation study of continuously administered regorafenib (BAY 73-4506), an inhibitor of oncogenic and angiogenic kinases, in patients with advanced solid tumors. J Clin Oncol.2010;28(15 Suppl):3035. 
20. Sunakawa Y, Furuse J, Okusaka T, et al. Regorafenib in Japanese patients with solid tumors: phase I study of safety, efficacy, and pharmacokinetics. Invest New Drugs. 2013;32(1):104-112.

21. Schultheis B, Folprecht G, Kuhlmann J, et al. Regorafenib in combination with FOLFOX or FOLFIRI as first- or second-line treatment of colorectal cancer: results of a multicenter, phase Ib study. Ann Oncol. 2013;24(6):1560-1567.

22. Argilés G, Troiani T, Rivera F, et al. First-line treatment with regorafenib (REG) in combination with mFOLFOX6 (folinic acid+5-fluorouracil [5-FU]+oxaliplatin) for metastatic colorectal cancer (mCRC): a singlearm, open-label phase II clinical trial. In: The 17th European Cancer Congress (ECCO); September 27, 2013; Amsterdam.

23. Grothey A, Van Cutsem E, Sobrero A, et al. Regorafenib monotherapy for previously treated metastatic colorectal cancer (CORRECT): an international, multicentre, randomised, placebo-controlled, phase 3 trial. Lancet. 2013;381(9863):303-312.

24. Yoshino T, Komatsu Y, Yamada Y, et al. Randomized phase III trial of regorafenib in metastatic colorectal cancer: analysis of the CORRECT Japanese and non-Japanese subpopulations. Invest New Drugs. 2015;33(3):740-750.

25. Li J, Qin S, Xu R, et al. Regorafenib plus best supportive care versus placebo plus best supportive care in Asian patients with previously treated metastatic colorectal cancer (CONCUR): a randomised, doubleblind, placebo-controlled, phase 3 trial. Lancet Oncol. 2015;16(6): 619-629.

26. Tougeron D, Desseigne F, Etienne PL, et al. REBECCA: a large cohort study of regorafenib (REG) in the real-life setting in patients (pts) previously treated for metastatic colorectal cancer (mCRC). Ann Oncol. 2014;25(Suppl 4):iv167-iv209; Abstr 6220 ESMO2014.

27. Van Cutsem E, Ciardiello F, Seitz J-F, et al. LBA-05 Results from the large, open-label phase $3 \mathrm{~b}$ CONSIGN study of regorafenib in patients with previously treated metastatic colorectal cancer. AnnOncol. 2015; 26(Suppl 4):iv118.

28. Grothey A, Van Cutsem E, Sobrero AF, et al. Time course of regorafenibassociated adverse events in the phase III CORRECT study. $J$ Clin Oncol.2012; 30(Suppl 34):467.

29. Falcone A, Van Cutsem E, Sobrero A, et al. Regorafenib dose modifications in patients with metastatic colorectal cancer in the phase III CORRECT study. In: The 17th European Cancer Congress (ECCO); September 27, 2013; Amsterdam.

30. Sueda T, Sakai D, Kudo T, et al. Efficacy and safety of regorafenib or TAS-102 in patients with metastatic colorectal cancer refractory to standard therapies. Anticancer Res. 2016;36(8):4299-4306.

31. Moriwaki T, Fukuoka S, Taniguchi H, et al. Propensity score analysis of regorafenib versus trifluridine/tipiracil in patients with metastatic colorectal cancer refractory to standard chemotherapy (REGOTAS): a Japanese society for cancer of the colon and rectum multicenter observational study. Oncologist. Epub 2017 Sep 11.

32. Tabchi S, Ghosn M. Regorafenib: start low and go slow. Target Oncol. 2015;10(3):445-447.

33. Grothey A. Regorafenib in metastatic colorectal cancer: optimal dosing and patient selection recommendations. Clin Adv Hematol Oncol.2015;13(8):514-517.

34. Siena S, Grothey A, Sobrero A, et al. Effects of regorafenib therapy on health-related quality of life in patients with metastatic colorectal cancer in the phase III CORRECT study. In: The 17th European Cancer Congress (ECCO); September 27, 2013; Amsterdam.

35. Chang J, Pawar V, Grothey A, et al. Time to health status deterioration in regorafenib-treated patients with metastatic colorectal cancer (mCRC): a post-hoc analysis of the phase III CORRECT study. In: The 17th European Cancer Congress (ECCO); September 27, 2013; Amsterdam.

36. Seal BS. Cost-effectiveness of regorafenib for pretreated metastatic colorectal cancer patients in the United States. J Clin Oncol 2012;30(Suppl 34):578.
37. Goldstein DA, Ahmad BB, Chen Q, et al. Cost-effectiveness analysis of regorafenib for metastatic colorectal cancer. J Clin Oncol. 2015;33(32):3727-3732.

38. Choi H, Charnsangavej C, Faria SC, et al. Correlation of computed tomography and positron emission tomography in patients with metastatic gastrointestinal stromal tumor treated at a single institution with imatinib mesylate: proposal of new computed tomography response criteria. J Clin Oncol.2007;25(13):1753-1759.

39. Eisenhauer EA, Therasse P, Bogaerts J, et al. New response evaluation criteria in solid tumours: revised RECIST guideline (version 1.1). Eur J Cancer 2009;45(2):228-247.

40. Marom EM, Martinez CH, Truong MT, et al. Tumor cavitation during therapy with antiangiogenesis agents in patients with lung cancer. J Thorac Oncol.2008;3(4):351-357.

41. Ricotta R, Sartore-Bianchi A, Verrioli A, Vanzulli A, Siena S. Regorafenib for metastatic colorectal cancer. Lancet. 2013;381(9877):1537.

42. Ricotta R, Verrioli A, Ghezzi S, et al. Radiological imaging markers predicting clinical outcome in patients with metastatic colorectal carcinoma treated with regorafenib: post-hoc analysis of the CORRECT phase III trial (RadioCORRECT study). ESMO Open. 2016;1(6):e000111.

43. Lim Y, Han SW, Yoon JH, et al. Clinical implication of anti-angiogenic effect of regorafenib in metastatic colorectal cancer. PLoS One. 2015;10(12):e0145004.

44. Adenis A, de la Fouchardiere C, Paule B, et al. Survival, safety, and prognostic factors for outcome with Regorafenib in patients with metastatic colorectal cancer refractory to standard therapies: results from a multicenter study (REBECCA) nested within a compassionate use program. BMC cancer. 2016;16:412.

45. Calcagno F, Lenoble S, Lakkis Z, et al. Efficacy, safety and cost of regorafenib in patients with metastatic colorectal cancer in French clinical practice. Clin Med Insights Oncol. 2016;10:59-66.

46. Del Prete S, Cennamo G, Leo L, et al. Adherence and safety of regorafenib for patients with metastatic colorectal cancer: observational real-life study. Future Oncol. 2017;13(5):415-423.

47. Gotfrit J, Vickers M, Sud S, et al. Real-life treatment of metastatic colorectal cancer with regorafenib: a single-centre review. Curr Oncol. 2017;24(4):234-239.

48. Kopeckova K, Buchler T, Bortlicek Z, et al. Regorafenib in the reallife clinical practice: data from the Czech registry. Target Oncol. 2017;12(1):89-95.

49. Padhani AR, Leach MO. Antivascular cancer treatments: functional assessments by dynamic contrast-enhanced magnetic resonance imaging. Abdom. Imaging. 2005;30(3):324-341.

50. Mross K, Fasol U, Frost A, et al. DCE-MRI assessment of the effect of vandetanib on tumor vasculature in patients with advanced colorectal cancer and liver metastases: a randomized phase I study. $J$ Angiogenes Res. 2009;1:5.

51. Eschbach RS, Clevert DA, Hirner-Eppeneder H, et al. Contrast-enhanced ultrasound with VEGFR2-targeted microbubbles for monitoring regorafenib therapy effects in experimental colorectal adenocarcinomas in rats with DCE-MRI and immunohistochemical validation. PLoS One. 2017;12(1):e0169323.

52. Komori A, Taniguchi H, Hamauchi S, et al. Serum CA19-9 response is an early predictive marker of efficacy of regorafenib in refractory metastatic colorectal cancer. Oncology. Epub 2017 Sep 2.

53. Suenaga M, Mashima T, Kawata N, et al. Serum VEGF-A and CCL5 levels as candidate biomarkers for efficacy and toxicity of regorafenib in patients with metastatic colorectal cancer. Oncotarget. 2016;7(23):34811-34823.

54. Tabernero J, Lenz HJ, Siena S, et al. Analysis of circulating DNA and protein biomarkers to predict the clinical activity of regorafenib and assess prognosis in patients with metastatic colorectal cancer: a retrospective, exploratory analysis of the CORRECT trial. Lancet Oncol. 2015;16(8): 937-948. 
55. Dressman D, Yan H, Traverso G, Kinzler KW, Vogelstein B. Transforming single DNA molecules into fluorescent magnetic particles for detection and enumeration of genetic variations. Proc Natl Acad Sci USA. 2003;100(15):8817-8822.

56. Diehl F, Li M, Dressman D, et al. Detection and quantification of mutations in the plasma of patients with colorectal tumors. Proc Natl Acad Sci USA. 2005;102(45):16368-16373.
57. Maeda A, Ando H, Ura T, et al. Association between ABCG2 and SLCO1B1 polymorphisms and adverse drug reactions to regorafenib: a preliminary study. Int J Clin Pharmacol Ther. 2017;55(5):409-415.

58. Chen D, Wei L, Yu J, Zhang L. Regorafenib inhibits colorectal tumor growth through PUMA-mediated apoptosis. Clin Cancer Res.2014;20(13):3472-3484.
Cancer Management and Research

\section{Publish your work in this journal}

Cancer Management and Research is an international, peer-reviewed open access journal focusing on cancer research and the optimal use of preventative and integrated treatment interventions to achieve improved outcomes, enhanced survival and quality of life for the cancer patient The manuscript management system is completely online and includes

\section{Dovepress}

a very quick and fair peer-review system, which is all easy to use. Visit http://www.dovepress.com/testimonials.php to read real quotes from published authors. 